\title{
A free boundary problem with curvature
}

\author{
Inwon C. Kim \\ Department of Mathematics, MIT*
}

July 28,2004

\begin{abstract}
In this paper we are interested in a free boundary problem whith a motion law involving the mean curvature term of the free boundary. Viscosity solutions are introduced as a notion of global-time solutions past singularities. We show the comparison principle for viscosity solutions, which yields the existence of minimal and maximal solutions for given initial data. We also prove uniqueness of the solution for several classes of initial data and discuss the possibility of nonunique solutions.
\end{abstract}

\section{$0 \quad$ Introduction}

In this paper we introduce a method to study existence and uniqueness properties of viscosity solutions for the free boundary problems of the following form:

$\left(P_{0}\right)$

$$
\left\{\begin{array}{l}
u_{t}-F\left(x, t, D u, D^{2} u\right)=0 \quad \text { in }\{u>0\} \\
V=G\left(n, \frac{\partial u}{\partial n}, \kappa\right)
\end{array}\right.
$$

Here $V$ is the normal velocity of the free boundary $\partial\{u>0\}$ at $(x, t), n$ is the inward normal vector of $\Gamma_{t}:=\partial\{u(\cdot, t)>0\}$ at $x$ with respect to $\{u(\cdot, t)>0\}$ and $\kappa$ is the mean curvature of $\Gamma_{t}$ at $x$ (taken positive if $\Gamma_{t}$ is convex in the direction of $n$.) We assume that (i) $F$ and $G$ are continuous, (ii) $F$ is uniformlly elliptic and (iii) $G$ is increasing in $\frac{\partial u}{\partial n}$ and decreasing in $\kappa$.

*77 Massachusetts Avenue, Cambridge, MA 02139. e-mail: ikim@math.mit.edu 
A two phase version of $\left(P_{0}\right)$ with $F=F\left(D^{2} u\right)=\Delta u$ and with $G$ depending also on $(x, t)$ (see remark) has been introduced in a recent paper [ACS], where the authors prove that Lipschitz free boundaries of viscosity solutions are $C^{1, \alpha}$.

To keep the idea simple we investigate a special case of $\left(P_{0}\right)$ :

$$
\begin{cases}u_{t}-\Delta u=0 & \text { in }\{u>0\} \\ V=\partial u / \partial n(x, t)-\kappa & \text { on } \partial\{u>0\}\end{cases}
$$

An elliptic version of $(P)$ was introduced in $[\mathrm{ACKS}]$ as a minimizing problem for the energy function

$$
E(v)=\int_{D}|D v|^{2}+\text { Area }\{v=0\}
$$

in a given domain $D$.

The purpose of this paper is to prove the global-time existence and uniqueness of the viscosity solution of (P). Typically for viscosity solutions, the main tool to derive such results is the comparison principle: Roughly speaking, viscosity solutions are defined by comparison with classical solutions of (P) in local domains (see Definition 1.1). Observe that if $u$ and $v$ are smooth solutions of $(\mathrm{P})$ then the comparison principle holds: in other words if $u<v$ in $\overline{\{u>0\}}$ at $t=0$, then $u \leq v$ for $t>0$. The main theorem of this paper says that the comparison principle also holds between two viscosity solutions of $(\mathrm{P})$. It follows that for a given initial data there exists the maximal and minimal viscosity solutions of $(\mathrm{P})$. Uniqueness of the viscosity solution only holds with additional conditions on initial data. In fact nonuniqueness is expected to occur in general setting, which will be discussed in the last section.

The difficulty in the analysis lies in the free boundary condition. Let us explain this more precisely with the following two examples. (a) Without the curvature term on the free boundary condition (P) becomes the Stefan problem, for which sub- and inf- convolutions were used to prove the comparison principle (see $[\mathrm{K} 1]$ ). The advantage of these convolutions is that they provide a smooth approximation of the free boundary with appropriate 'first-order' information: roughly speaking, when the free boundaries of these two convolutions contact each other, it follows from the definition that both free boundaries are 'almost' $C^{1}$ in space and time at the contact point, and hence this helps estimating first order terms of the solutions near the contact point. Nevertheless we still need better approximations for second-order terms such as the mean curvature term $\kappa$ in $(\mathrm{P})$. (b) On the other hand, without the gradient term on the free boundary condition the two equations in $(\mathrm{P})$ are decoupled and the evolution of the free boundary is the mean curvature flow: $V=-\kappa$. For the mean curvature flow Evans and Spruck [ES] have proved the comparison principle by another kind of convolutions, which has been used in standard 
viscosity solutions arguments for fully nonlinear PDEs (see [CL], [CIL].) These convolutions do provide good estimates for the second order terms, but they might approach boundary points from interior points and vice versa. This becomes a problem in our case since we have two different equations on the boundary and in the positive set.

Therefore it is necessary to develop a new approach to study viscosity solutions of $(\mathrm{P})$ or more generally free boundary problems with second order terms. In the following sections we combine both approaches discussed in (a)-(b) to obtain our results. In section 1 we introduce the basic notions and define a notion of viscosity solutions. It can be easily checked that the definition we give in this paper is equivalent to that of [ACS]. In section 2 we prove the comparison principle. In section 3 existence and uniqueness results are proven based on the comparison principle.

\section{Remark}

1. In two-phase version of $\left(P_{0}\right)$ our proof for the comparison principle (Theorem 1.2) between viscosity solutions still holds. However in this case it is not clear to the author how to construct a supersolution and a subsolution which agrees with the given initial data at $t=0$ to apply Perron's method for the existence result (see the proof of theorem 3.1.).

2. Our method extends to the case when $G$ has $(x, t)$-dependence if one adds additional regularity assumption on $G$ in $(x, t)$-variable, such as uniform Lipschitz continuity of $G$ in $(x, t)$-variable independent of other variables. The difficulty lies in that the sup- and inf-convolutions introduced in $(2.1)-(2.2)$ does not preserve $\left(P_{0}\right)$ in general.

\section{Notations and Main result}

First we introduce several notations used in this paper:

- (a) $D(x ; r):=\left\{y \in \mathbb{R}^{n}:|x-y|<r\right\}$ for $x \in \mathbb{R}^{n}$.

(b) $B(x, t ; r)=\left\{(y, s) \in \mathbb{R}^{n+1}:|(x, t)-(y, s)|<r\right\}$ for $(x, t) \in \mathbb{R}^{n+1}$.

Given $B=B(x, t ; r)$, we denote $k B$ as a ball with the same center as $B$ and radius $k r$. Similarly we define $k D$ for $D=D(x ; r)$.

- Let $u, v: Q=\mathbb{R}^{n} \times[0, \infty) \rightarrow \mathbb{R}$ and let $D \subset Q$.

(a) $u$ is touching $v$ from below at $P_{0}$ in $D$ if $u-v$ has a local maximum zero at $P_{0}$ in a parabolic neighborhood of $P_{0}$ in $D$.

(b) $u$ is touching $v$ from above at $P_{0}$ in $D$ if $u-v$ has a local minimum zero at $P_{0}$ in a parabolic neighborhood of $P_{0}$ in $D$. 
- For $u: Q \rightarrow[0, \infty)$,

$$
\begin{aligned}
& \text { (i) } u^{*}(x, t):=\lim \sup _{(y, s) \rightarrow(x, t)} u(y, s) \text {; } \\
& \text { (ii) } u_{*}(x, t)=\liminf (y, s) \rightarrow(x, t) \\
& \text { in }
\end{aligned}
$$

- For $u: Q \rightarrow[0, \infty), x \in \mathbb{R}^{n}$ and $t \in[0, \infty)$ we denote

$$
\begin{gathered}
\Gamma(u)=\partial\{u>0\} ; \quad \Gamma_{t}(u)=\{x:(x, t) \in \Gamma(u)\} . \\
\Omega(u)=\{u>0\} ; \quad \Omega_{t}(u)=\{u(\cdot, t)>0\} .
\end{gathered}
$$

When $\Gamma_{t}(u)$ is $C^{2}$, let $\kappa_{p_{0}}\left(\Gamma_{t}(u)\right)$ be the mean curvature of $\Gamma_{t}(u)$ at $p_{0}$, taken positive if $\Gamma_{t}(u)$ is convex with respect to $\Omega_{t}(u)$.

- We say that a pair of functions $u_{0}, v_{0}$ is strictly ordered $\left(u_{0} \prec v_{0}\right)$ if

(i) $\operatorname{supp}\left(u_{0}\right)=\overline{\left\{u_{0}>0\right\}}$ is bounded, and it satisfies

$$
\operatorname{supp}\left(u_{0}(x)\right) \subset \operatorname{Int}\left(\operatorname{supp}\left(v_{0}(x)\right)\right)
$$

(ii) inside $\operatorname{supp}\left(u_{0}\right)$ the functions are strictly ordered:

$$
u_{0}(x)<v_{0}(x)
$$

- We define $\mathcal{S}(n)$ to be the set of symmetric $n \times n$ matrices. For $X, Y \in \mathcal{S}(n), X \leq Y$ means that

$$
v X v^{T} \leq v Y v^{T} \text { for any vector } v \in \mathbb{R}^{n}
$$

Next we define viscosity sub- and supersolutions of $(\mathrm{P})$ for a given initial data $u_{0}: \mathbb{R} \rightarrow$ $[0, \infty)$.

Definition 1.1 (a) A nonnegative upper-semicontinuous function $u$ in $Q$ is a viscosity subsolution of $(P)$ with initial data $u_{0}$ if (1) $u(x, 0)=u_{0}$, (2) $\overline{\Omega(u)} \cap\{t=0\}=\bar{\Omega}\left(u_{0}\right)$ and (3) for any $C^{2,1}$-function $\phi$ which touches $u$ from above at $(x, t)$ in $\bar{\Omega}(u)$, the following holds:

(i) $\phi_{t}-\Delta \phi(x, t) \leq 0$ if $u(x, t)>0$;

(ii) $\min \left(\phi_{t}-\Delta \phi, \phi_{t}-|D \phi|^{2}+\kappa|D \phi|\right)(x, t) \leq 0$ if $u(x, t)=0$ and $|D \phi|(x, t)>0$; 
where $\kappa$ denotes $\kappa_{x}\left(\Gamma_{t}(\phi)\right)$.

(b) A nonnegative lower-semicontinuous function $v$ in $Q$ is a viscosity supersolution of $(P)$ with initial data $u_{0}$ if (1) $v(x, 0)=u_{0}$, (2) $\overline{\Omega(v) \cap\{t>0\}} \cap\{t=0\} \supseteq \bar{\Omega}\left(u_{0}\right)$ and (3) for any $C^{2,1}$-function $\psi$ which touches $v$ from below at $(x, t)$ in $\bar{\Omega}(v)$, the following holds:

(i) $\psi_{t}-\Delta \psi(x, t) \leq 0$ if $v(x, t)>0$;

(ii) $\max \left(\psi_{t}-\Delta \psi, \psi_{t}-|D \psi|^{2}+\kappa|D \psi|\right)(x, t) \geq 0$ if $v(x, t)=0$ and $|D \psi|(x, t)>0$.

We define $u$ to be a viscosity solution of $(\mathrm{P})$ if $u=u_{*}$ is a (viscosity) supersolution and $u^{*}$ is a subsolution of $(\mathrm{P})$. Below we state our main theorem in this paper:

Theorem 1.2 (comparison principle) Let $u$ and $v$ be respectively a sub-and supersolution of $(P)$ with strictly separated initial data, $u_{0} \prec v_{0}$. Then the solutions remain ordered for all time:

$$
u(\cdot, t) \prec v(\cdot, t) \quad \text { for } t>0 .
$$

\section{Proof in the case of smooth solutions:}

If $u, v$ and their free boundaries are smooth ( $C^{2}$ in space-time), then theorem 1.2 can be proven easily as follows: Let $t_{0}$ to be the first time $u$ touches $v$ from below, that is

$$
t_{0}=\sup \{t: u(\cdot, t) \prec v(\cdot, t)\} .
$$

From the maximal principle for heat equations, it follows that then $\Gamma(u)$ and $\Gamma(v)$ touches for the first time at $P_{0}:=\left(x_{0}, t_{0}\right)$.We call such point $\left(x_{0}, t_{0}\right)$ as the 'contact point' of $u$ and $v$. Since $u \leq v$ at $t=t_{0}$ and $\Gamma_{t_{0}}(u)$ touches $\Gamma_{t_{0}}(v)$ with $\Gamma_{t_{0}}(u) \subset \bar{\Omega}_{t_{0}}(v)$, it follows that

$$
\frac{\partial u}{\partial n}<\frac{\partial v}{\partial n}
$$

and

$$
-\kappa_{1}:=-\kappa_{x_{0}}\left(\Gamma_{t_{0}}(u)\right) \leq-\kappa_{x_{0}}\left(\Gamma_{t_{0}}(v)\right):=-\kappa_{2}
$$

where $n$ is the inward normal vector of $\Gamma_{t_{0}}(u)$ (and $\Gamma_{t_{0}}(v)$ ) at $x_{0}$, and $\kappa_{x}\left(\Gamma_{t}\right)$ denotes the mean curvature of $\Gamma_{t}$ at $x$. ((1.1) is due to Hopf's lemma and the regularity of $\Gamma_{t}(u)$.) On the other hand by the definition of contact point, $\Gamma(u)$ has to 'touch' $\Gamma(v)$ for the first time at $\left(x_{0}, t_{0}\right)$, 
and thus $\Gamma(u)$ propagates faster than $\Gamma(v)$ at $\left(x_{0}, t_{0}\right)$ in the normal direction. Since $\Omega(u)$ and $\Omega(v)$ are respectively the positive set of $u$ and $v$, the normal velocity of $\Gamma(u)$ and $\Gamma(v)$ are equal to $u_{t} /|D u|$ and $v_{t} /|D v|$ respectively, and thus

$$
\frac{u_{t}}{|D u|} \geq \frac{v_{t}}{|D v|} \text { at }\left(x_{0}, t_{0}\right)
$$

This leads to a contradiction since

$$
\frac{u_{t}}{|D u|} \leq|D u|-\kappa_{1}<|D v|-\kappa_{2} \leq \frac{v_{t}}{|D v|} \text { at }\left(x_{0}, t_{0}\right)
$$

due to the free boundary condition.

For nonsmooth solutions we pursue above arguments by a series of approximations as summarized below:

1. First we introduce sup- and inf- convolutions $Z$ and $W$, respectively of $u$ and $v$, for which the mean curvature and the gradient terms in $(\mathrm{P})$ are bounded.

2. Next we use the main lemma of [CIL] to approximate the contact point $P_{0}$ by nearby pairs of 'regular' points $\left(P^{\epsilon}, Q^{\epsilon}\right)$ respectively on $\bar{\Omega}(Z)$ and $\bar{\Omega}(W)$. At these regular points $Z$ and $W$ can be approximated by smooth $C^{2,1}$ functions. Here we consider the characteristic functions of $\Omega(Z)$ and $\Omega(W)$ instead of the original functions to ensure that the 'regular' points lay on the free boundary of $Z$ and $W$ (otherwise we cannot argue as in (1.4)). Note that by taking characteristic functions we preserved geometric quantaties of the free boundary such as mean curvature $\kappa$ or the normal velocity $V$. Roughly speaking by this process we have obtained smooth hypersurfaces respectively approximate to $\Gamma(Z)$ and $\Gamma(W)$ in small neighborhoods of $P^{\epsilon}$ and $Q^{\epsilon}$.

3. Based on these hypersurfaces, next we construct the barrier functions for $Z$ and $W$ to compare $D Z\left(P^{\epsilon}\right)$ and $D W\left(Q^{\epsilon}\right)$ as $P^{\epsilon}, Q^{\epsilon} \rightarrow P_{0}$. Here we strongly use the interior- and exteriorball property (see section 2) of $Z$ and $W$.

4. Finally to prove theorem 1.2 we send $\epsilon \rightarrow 0$ and argue as in (1.1)-(1.4) to obtain a contradiction. 


\section{Comparison Principle}

\section{Regularizing the free boundary}

We begin with inf- and sup-convolutions which are used, for instance, in [K2]. For given $r>\gamma>0$ let us define

$$
\begin{gathered}
Z(x, t)=\sup _{B(x, t ; r)} U(y, s) \quad \text { where } U(x, t)=\sup _{D(x ; r)} u(y, t), \\
W(x, t)=\inf _{B(x, t ; r-\gamma t)} V(y, s) \quad \text { where } V(x, t)=\inf _{D(x ; r)} v(y, t) .
\end{gathered}
$$

for $r \leq t \leq r / 2 \gamma$. We mention that we apply the convolutions twice in above definitionof $Z$ and $W$ to guarantee the semi-finite speed of propagation property of $\Gamma(Z)$ and $\Gamma(W)$ (see Lemma 1.3). From the definitions it is not hard to check that $Z, U$ and $W, V$ are respectively viscosity sub- and supersolution of $(P)$. Indeed $W$ is a 'strict' supersolution of $(P)$ in the sense that it satisfies

$$
W_{t} /|D W|=|D W|-\kappa+\gamma \text { on } \Gamma(W) \text { for } r \leq t<r / 2 \gamma
$$

in the viscosity sense: that is, in the context of Definition 1.1.

Since $u_{0} \prec v_{0}$ and $\bar{\Omega}(u) \cap\{t=0\}=\overline{\left\{u_{0}>0\right\}}$, we can take $r>0$ small enough that $Z \prec W$ at $r \leq t \leq 2 r$. For such $r>0$ and $0<\gamma<<r$ we would like to show that

$$
Z(\cdot, t) \prec W(\cdot, t) \quad \text { for } r<t<r / 2 \gamma \text {. }
$$

Then by sending $\gamma$ to zero in (2.3), it follows that $u \prec v$ for $t>0$.

Before proving our claim, we observe some properties of $Z$ and $W$ :

\section{Interior and exterior ball property}

Let $\Omega$ be a bounded domain in $Q$. We say that $\Omega$ has the interior (exterior) ball property at $P=(x, t) \in \partial \Omega$ if there is a closed $n+1$ dimensional (space-time) ball $B \subset \Omega$ (resp. $\Omega^{c}$ ) such that $B \cap \overline{\Omega^{c}}$ (resp. $\left.\bar{\Omega}\right)=P$. We then say that $B$ is interior (exterior) to $\Omega$ at $P$. Similarly we define interior(exterior) ellipsoid property. 


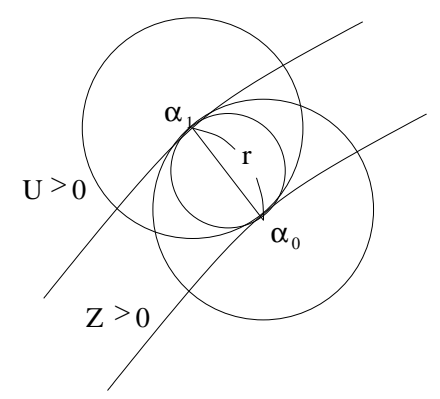

Figure 1.

From definition of $Z$ and $W$ it follows that on $\Gamma(Z)($ resp. $\Gamma(W))$ the positive set $\Omega(Z)$ ( resp. $\Omega(W)$ ) has the interior ball ( resp. exterior ellipsoid ) property. For example, at $\alpha_{0} \in \Gamma(Z)$, the set $\Omega(Z)$ has an interior space-time ball of radius $r$, centered at $\alpha_{1} \in \Gamma(U)$, while at $\alpha_{1}$ the set $\{U>0\}$ has an exterior space-time ball of radius $r$ at $\alpha_{0}$ (see figure 1.)

\section{Semi-finite propagation property}

At $P \in \Gamma(Z)$, consider the tangent hyperplane $H$ to the interior ball of $\Omega(Z)$ at $P$. The inward normal vector to $H$ with respect to $\Omega(Z)$ at $P$ can be written as $\left(\nu, m_{P}\right)$ where $\nu$ is a unit vector in $\mathbb{R}^{n}$ and $-\infty \leq m_{P} \leq \infty$, where $(\nu, \pm \infty)$ denotes the vector $(0, \pm 1), 0 \in \mathbb{R}^{n}$. We then call $m_{P}$ as the advancing speed (or slope) of $\Gamma(Z)$ at $P$. Similarly one can define the advancing speed $n_{Q}$ of $\Gamma(W)$ at $Q$.

Lemma 2.1 Let $m_{P}, n_{Q}$ be as defined above. Then $m_{p}<\infty, n_{q}>-\infty$ for $t \geq 2 r$.

\section{Proof.}

1. Suppose $m_{P}=\infty$. Then by definition there is a ball $B_{1}$ of radius $r / 2$ and $P_{1} \in \Gamma(U)$ such that

(i) $B_{1}$ is exterior to $\{U>0\}$ at $P_{1}$

(ii) $\overline{P P_{1}}=(0, \ldots, 0, r)$ (see figure 2.)

By definition of $U$ there exists $p_{1}=\left(x_{1}, t_{1}\right) \in \Gamma(u)$ where $\left|p_{1}-P_{1}\right|=r$. Moreover $u=0$ in the region $L_{1}=\left\{(y, s):|y-x|=r,(x, s) \in B_{1}\right\}$. In particular at $p_{1}$, the set $\Omega(u)$ has an exterior ball $B_{1}^{\prime}$ : a translate of $B_{1}$ with horizontal tangency and the set $\Omega_{t_{1}}(u)$ has an exterior disk $D_{1}=D\left(P_{1} ; r\right)$ ( see figure 3.)

Observe that $2 D_{1} \subset L_{1} \cap\left\{t=t_{1}-r\right\}$. For given $\epsilon>0$ let us consider the domain

$$
\Sigma=\left\{(x, t): x \in\left(2 D_{1}-L_{2}(t)\right), t \in\left(t_{1}-r, t_{1}\right)\right.
$$




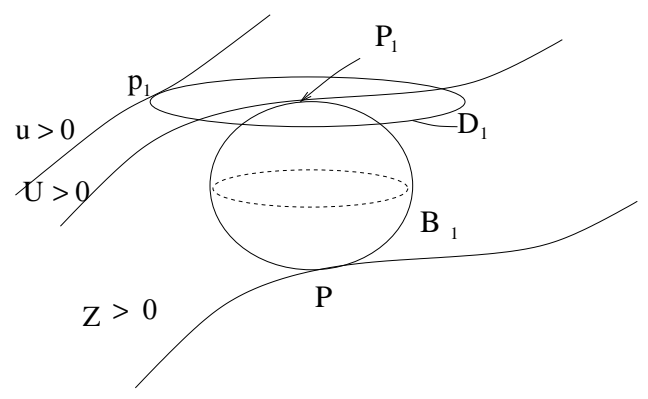

Figure 2.

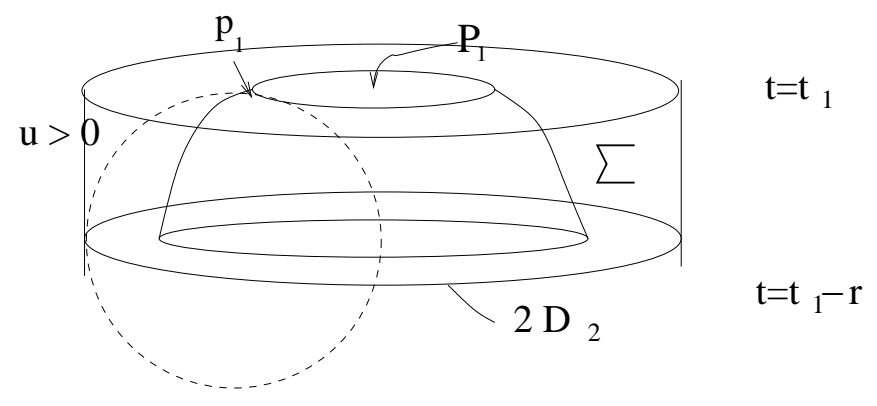

Figure 3.

where $L_{2}(t)=(1+f(t)) D_{1}$ where $f(t)=\sqrt{\left(t_{1}-t\right)} / 2$ for $t \in\left(t_{1}-r, t_{1}-\epsilon\right), f\left(t_{1}\right)=0$ and $f$ is $C^{1}$ and $f^{\prime}$ is monotone decreasing in $\left[t_{1}-r, t_{1}\right]$.

Note that $L_{2}(t)$ lies outside of $\bar{\Omega}(u)$ for $t \in\left(t_{1}-r, t_{1}\right)$. (see figure 3.) Next we consider the function $\omega$ in $\Sigma$ satisfying

$$
\begin{cases}-\Delta \omega=1 & \text { in } \Sigma, \\ \omega=\sup _{\Sigma} u & \text { on } \partial 2 D_{1} \\ \omega=0 & \text { on } \partial L_{2}(t)\end{cases}
$$

Since $\omega(x, t)$ is increasing,

$$
\omega_{t}-\Delta \omega>0 \text { in } \Sigma
$$

Note that $|D \omega|$ on $\Gamma(\omega)$ is uniformly bounded in $\Sigma$ only depending on $r$ and on the supremum of $u$ on $\Sigma$ because of the exterior disk condition. Moreover the mean curvature $\kappa$ of $\Gamma(\omega)$ at $p_{1}$ is $-1 / r$. Therefore if we choose $\epsilon$ small enough, then 


$$
\omega_{t} /|D \omega|+\kappa \geq \sqrt{r / \epsilon}-1 / r>|D \omega| \text { on } \Gamma_{t_{1}}(\omega)
$$

and therefore $\omega$ is a supersolution of $(P)$ near $t=t_{1}$. Moreover $\omega$ is $C^{2,1}$ in $\Sigma$.

Note that $u=0 \leq \omega$ at $t=t_{1}-r$. Furthermore $u \leq \omega$ on $\partial 2 D_{2}$ and $u=0$ on $L_{2}(t)$ for $t \in\left(t_{1}-r, t_{1}\right)$. Therefore it follows from (2.4) that $u \leq \omega$ for $t<t_{1}$ and $u$ touches $\omega$ from below at $t=t_{1}$, which contradicts (2.4)-(2.5). Hence we obtain $m_{P}<\infty$.

3. Next suppose that $n_{Q}=-\infty$. Then by definition of $W$ there is a ball $B_{1}$ of radius $r / 4$ and $Q_{2} \in \Gamma(V)$ such that

(i) $B_{1}$ is interior to $\Omega(V)$ at $Q_{2}$.

(ii) $\overline{P P_{2}}=(0, \ldots, 0, r)$.

By definition, $v>0$ in the region $L_{2}=\left\{(y, s):|y-x|=1,(x, s) \in B_{1}\right\}$. In particular at $p_{2} \in \Gamma(v)$, the set $\Omega(v)$ has an interior ball $B_{1}^{\prime}$ : a translate of $B_{1}$ with horizontal tangency and the set $\Omega_{t_{2}}(v)$ has an interior disk $D_{1}$ with center $P_{2}$. A parallel argument as in the previous steps, by constructing a test function in a parabolic neighborhood of $p_{2}$, leads to a contradiction.

\section{Remark}

Indeed for any $T<\infty$, from the proof Lemma 2.1 indicates that $m_{P} \leq M$ for $P \in \Gamma(Z) \cap\{t \leq T\}$, where $M$ depends on $r$ and the supremum of $u$ for $t \leq T+r$. Similarly one can also check that $n_{Q} \geq-N$ for $Q \in \Gamma(W) \cap\{t \leq T\}$ where $N$ depends on $r$ and the infimum of $v(x, t)$ where $t \leq T+r$ and $(x, t) \in \Omega(v)$ is more than $r$-away from $\Gamma(v)$.

\section{Perturbation of the contact point}

From now on we fix $r>0$ and begin our analysis on $Z$ and $W$. Suppose that the claim (2.3) is not true. Then for $\gamma>0$ chosen small we have

$$
2 r \leq t_{0}=\sup \{Z(x, \tau) \prec W(x, \tau) \quad \text { for } r \leq \tau<t\}<r / 2 \delta .
$$

By Lemma 1.3 it follows that there is a point $P_{0}=\left(x_{0}, t_{0}\right)$ where $\Gamma(Z)$ intersects with $\Gamma(W)$ for the first time. Moreover observe that $n_{P_{0}} \leq m_{P_{0}}$ by definition. Hence due to Lemma 2.1

$$
-\infty<n_{P_{0}} \leq m_{P_{0}}<\infty
$$


For any $\epsilon>0$ we consider

$$
\Phi_{\epsilon}(x, y, t)=\bar{Z}(x, t)-\bar{W}(y, t)-\frac{|x-y|^{2}}{2 \epsilon}-\frac{\epsilon}{\left(t_{0}-t\right)}
$$

where $\bar{Z}=\mathbb{1}_{\bar{\Omega}(Z)}-1$ and $\bar{W}=\mathbb{1}_{\Omega(W)}$. Observe that $\bar{Z}$ is upper-semicontinuous and $\bar{W}$ is lower-semicontinuous in $Q$. Also note that $\bar{Z}(x, t)-\bar{W}(x, t) \leq-1$ for $t<t_{0}$ and $\bar{Z}-\bar{W}=0$ at $\left(x_{0}, t_{0}\right)$. Thus for any $\epsilon>0$ there is $\left(\tilde{x}_{\epsilon}, \tilde{y}_{\epsilon}, \tilde{t}_{\epsilon}\right)$ where $\Phi_{\epsilon}(x, y, t)$ has a local maximum in $Q \cap\left[0, t_{0}\right)$.

Moreover due to (2.4) there exists $(x, t) \in \bar{\Omega}(Z)$ and $(y, t) \in\{W=0\}$ such that $t=t_{0}-2 \epsilon$ and $|x-y|^{2}<\epsilon / 4$. It follows that $\left(\tilde{x}_{\epsilon}, \tilde{t}_{\epsilon}\right) \in \bar{\Omega}(Z)$ and $\left(\tilde{y}_{\epsilon}, \tilde{t}_{\epsilon}\right) \in\{W=0\}$ with

$$
\left|\tilde{x}_{\epsilon}-\tilde{y}_{\epsilon}\right|=O\left(\epsilon^{1 / 2}\right)
$$

and $\tilde{t}_{\epsilon} \rightarrow t_{0}$. In particular we have

$$
\left(\tilde{x}_{\epsilon}, \tilde{y}_{\epsilon}, \tilde{t}_{\epsilon}\right) \rightarrow\left(x_{0}, x_{0}, t_{0}\right) \text { as } \epsilon \rightarrow 0 .
$$

Note that $\left|x_{\epsilon}-y_{\epsilon}\right|>0$ for each $\epsilon>0$ since $t_{\epsilon}<t_{0}$.

Next we apply theorem 8.3 in [CIL], which yields for $\epsilon>0$

$$
\left(\tilde{a}_{\epsilon}, \tilde{p}_{\epsilon}, \tilde{X}_{\epsilon}\right) \in \bar{P}^{2,+}(\bar{Z})\left(\tilde{x}_{\epsilon}, \tilde{t}_{\epsilon}\right) \text { and }\left(\tilde{b}_{\epsilon}, \tilde{q}_{\epsilon}, \tilde{Y}_{\epsilon}\right) \in \bar{P}^{2,-}(\bar{W})\left(\tilde{y}_{\epsilon}, \tilde{t}_{\epsilon}\right)
$$

with

$$
\tilde{p}_{\epsilon}=\tilde{q}_{\epsilon}=\left(\tilde{x}_{\epsilon}-\tilde{y}_{\epsilon}\right) / \epsilon \neq 0, \quad \tilde{X}_{\epsilon} \leq \tilde{Y}_{\epsilon} \quad \text { and } \tilde{a}_{\epsilon}-\tilde{b}_{\epsilon} \geq \epsilon /\left(t_{0}-\tilde{t}_{\epsilon}\right)^{2}
$$

Here $P^{2, \pm}(u)(x, t)$ denotes the 'parabolic superjet' and 'subjet' of $u$ at $(x, t)$ and $\bar{P}^{2, \pm}(u)(x, t)$ denotes their closures: For example, $(a, p, X) \in \mathbb{R} \times \mathbb{R}^{n} \times \mathcal{S}(n)$ lies in $P^{2,+}(\bar{Z})(x, t)$ if

$$
\bar{Z}(y, s) \leq \bar{Z}(x, t)+a(s-t)+<p, y-x>+\frac{1}{2}(y-x) X(y-x)^{T}+o\left(|s-t|+|y-x|^{2}\right)
$$

as $(y, s) \rightarrow(x, t)$. We refer to section 8 of [CIL] for the full definitions.

From the above result, it follows that for every $\epsilon>0$ we can pick (a) a pair of points $\left(x_{\epsilon}, t_{\epsilon}\right)$ and $\left(y_{\epsilon}, s_{\epsilon}\right)$ respectively on $\Gamma(Z)$ and on $\Gamma(W)$; (b) a small neighborhood $N_{1}^{\epsilon}$ and $N_{2}^{\epsilon}$ around each point; and (c) $a_{\epsilon}, b_{\epsilon} \in \mathbb{R}, p_{\epsilon}, q_{\epsilon} \in \mathbb{R}^{n}, X_{\epsilon}, Y_{\epsilon} \in \mathcal{S}(n)$ such that 

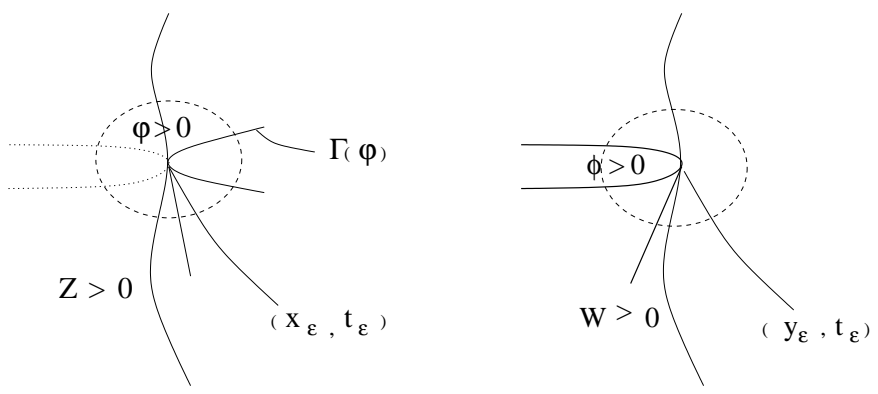

Figure 4.

$$
\begin{cases}(i) \quad \varphi^{\epsilon}(x, t):=\left[a_{\epsilon}\left(t-t_{\epsilon}\right)+p_{\epsilon} \cdot\left(x-x_{\epsilon}\right)+\frac{1}{2}\left(x-x_{\epsilon}\right) X_{\epsilon}\left(x-x_{\epsilon}^{T}\right)\right]_{+} \geq \bar{Z}(x, t) \text { in } N_{1}^{\epsilon} \\ (\text { ii }) \quad \phi^{\epsilon}(y, s):=\left[b_{\epsilon}\left(s-s_{\epsilon}\right)+q_{\epsilon} \cdot\left(y-y_{\epsilon}\right)+\frac{1}{2}\left(y-y_{\epsilon}\right) Y_{\epsilon}\left(y-y_{\epsilon}\right)^{T}\right]_{+} \leq \bar{W}(y, s) \text { in } N_{2}^{\epsilon} \\ (\text { iii }) \quad a_{\epsilon}-b_{\epsilon} \geq 0, X_{\epsilon}-Y_{\epsilon} \leq \epsilon\left|\tilde{p}_{\epsilon}\right| I \text { and } \\ & \left|p_{\epsilon}-\tilde{p}_{\epsilon}\right|,|| p_{\epsilon}|-| q_{\epsilon}||,\left|p_{\epsilon}-q_{\epsilon}\right| \leq \epsilon^{2} \min \left[1,\left|\tilde{p}_{\epsilon}\right|^{2}\right] \\ (\text { iv }) & x_{\epsilon}=\tilde{x}_{\epsilon}+O\left(\epsilon^{2}\right), y_{\epsilon}=\tilde{y}+O\left(\epsilon^{2}\right) \text { and } t_{\epsilon}, s_{\epsilon}=\tilde{t}_{\epsilon}+O(\epsilon) \\ \text { (v) } \quad Z\left(\cdot, t_{\epsilon}\right) \leq W\left(\cdot, s_{\epsilon}\right)+O\left(\epsilon^{2}\right)\end{cases}
$$

Remark (i) - (ii) means that $\left(a_{\epsilon}, q_{\epsilon}, X_{\epsilon}\right) \in P^{2,+}(\bar{Z})\left(x_{\epsilon}, t_{\epsilon}\right)$ and $\left(b_{\epsilon}, q_{\epsilon}, Y_{\epsilon}\right) \in P^{2,-}(\bar{W})\left(y_{\epsilon}, s_{\epsilon}\right)$. (iii)-(iv) means that for example $a_{\epsilon}, b_{\epsilon}$ are close enough to $\tilde{a}_{\epsilon}, \tilde{b}_{\epsilon}$ and so on. (v) is possible since $t_{\epsilon}, s_{\epsilon}$ is very close to $\tilde{t}_{\epsilon}, Z \leq W$ at $\tilde{t}_{\epsilon}<t_{0}$ and $Z-W$ is upper-semicontinuous.

From now on we will analyze these test functions $\phi^{\epsilon}$ and $\varphi^{\epsilon}$ and send $\epsilon$ to zero to derive a contradiction. There are two main difficulties involved. First of all, note that the test functions are comparable to the characteristic functions $\bar{Z}$ and $\bar{W}$, not to $Z$ and $W$. As a result what we obtained is smooth 'test sets' which are locally interior to $\Gamma(Z)$ and exterior to $\Gamma(W)$ (see figure 4.) Thus for our analysis on $Z$ and $W$ we need to construct a new pair of test functions $\left(\psi^{\epsilon}, \xi^{\epsilon}\right)$ based on the 'test sets' so that $\psi^{\epsilon}$ is comparable to $Z$ and $\xi^{\epsilon}$ is comparable to $W$. For technical reasons, instead of $Z$ and $W$ we consider $U$ and $V$ at the corresponding free boundary points. More precisely, we will construct the test functions so that $\psi^{\epsilon}$ is bigger than and comparable to $U$ and $\xi^{\epsilon}$ is less than and comparable to $V$ (see (2.14) and (2.16)).

Secondly since we would like to compare the gradient sizes of test functions $D \psi^{\epsilon}$ and $D \xi^{\epsilon}$ respectively at $\left(x_{\epsilon}, t_{\epsilon}\right)$ and $\left(y_{\epsilon}, s_{\epsilon}\right)$ using $(2.9)(\mathrm{v})$. Heuristically speaking, we would argue that for $\left(y, t_{\epsilon}\right)$ in a neighborhood of $\left(x_{0}, t_{0}\right)$, 


$$
\left|D \psi^{\epsilon}\right|\left(x_{\epsilon}, t_{\epsilon}\right) \sim \frac{Z\left(y, t_{\epsilon}\right)}{d\left(y, \Gamma_{t_{\epsilon}}(W)\right)} \leq \frac{W\left(y, s_{\epsilon}\right)+O\left(\epsilon^{2}\right)}{d\left(y, \Gamma_{s_{\epsilon}}(Z)\right)} \sim\left|D \psi^{\epsilon}\right|\left(y_{\epsilon}, s_{\epsilon}\right)+O(\epsilon)
$$

For above argument to hold, the test functions have to be smooth enough and comparable to $Z$ and $W$ respectively in domains $D_{1}$ and $D_{2}$. Also the domains should be big enough: for example we should be able to pick $\left(y, s_{\epsilon}\right) \in D_{2}$ such that $d\left(y, \Gamma_{s_{\epsilon}}(Z)\right)>>\epsilon^{2}$. Below we construct such a domain by extending $N_{i}^{\epsilon}, i=1,2$. (For technical reasons our test functions will be comparable to $U$ and $V$ instead ot $Z$ and $W$ (see (2.1)-(2.2) for definition)

\section{Extending domains}

Let us fix $\epsilon>0$ and let $e_{n}$ denote $p_{\epsilon} /\left|p_{\epsilon}\right|$ : the normal vector of $\Gamma_{t_{\epsilon}}\left(\varphi^{\epsilon}\right)$ at $x_{\epsilon}$ and let $x_{n}=$ $x \cdot e_{n}, x^{\prime}=\left(x_{1}, \ldots x_{n-1}\right)$. Observe that $e_{n}$ is also the inward spatial normal vector of the ball $B_{1}^{\epsilon}$ of radius $r$ which is interior to $\Omega(Z)$ and touches $\Gamma(Z)$ at $\left(x_{\epsilon}, t_{\epsilon}\right)$. For $0<C<1$ to be chosen later, there is a space-time ball $B_{2}^{\epsilon}$ of radius $C r$ which touches $\Gamma(U)$ at $\left(x_{\epsilon}^{1}, t_{\epsilon}^{1}\right)$ from outside and $B_{1}^{\epsilon}$ at $\left(x_{\epsilon}, t_{\epsilon}\right)$ has the same normal vector as $B_{2}^{\epsilon}$ at $\left(x_{\epsilon}, t_{\epsilon}^{1}\right)$. Let $D_{2}^{\epsilon}=B_{2}^{\epsilon} \cap\left\{t=t_{\epsilon}^{1}\right\}$. Due to (2.1), the hyperplane of the interior ball (with radius $r$ ) of $\Gamma(Z)$ at $\left(x_{0}, t_{0}\right)$ is not horizontal at the contact point $\left(x_{0}, t_{0}\right)$. Moreover it follows from this fact that the corresponding interior balls (with radius $r$ ) of $Z$ at $\left(x_{\epsilon}, t_{\epsilon}\right)$ cannot be horizontal if $\epsilon$ is small enough. (Otherwise the limiting space-time ball (with radius $r$ ) with horizontal tangency is an interior to $Z$ at $\left(x_{0}, t_{0}\right)$ which leads to a contradiction.)

Indeed the same argument shows that, the slope of $\Gamma(Z)$ at $\left(x_{\epsilon}, t_{\epsilon}\right)$ is uniformly bounded by $m+1$ and $m-1$ if $0<\epsilon<<1$, where $m$ is the slope of $\Gamma(Z)$ at $\left(x_{0}, t_{0}\right)$. Now it follows that $D_{2}^{\epsilon}$ has a positive radius $k r, 0<k<1 / 2$ where $k$ depends only on $m, r$ and thus independent of $\epsilon>0$.

Next we observe that $\Gamma\left(\varphi^{\epsilon}\right)$ has its slope $a_{\epsilon} /\left|p_{\epsilon}\right|$ at $\left(x_{\epsilon}, t_{\epsilon}\right)$, which is bigger than than $O(\epsilon)+b_{\epsilon} /\left|q_{\epsilon}\right|$ by $(2.9)$ (iii). But $b_{\epsilon} /\left|q_{\epsilon}\right|$ is the slope of $\Gamma\left(\phi^{\epsilon}\right)$ at $\left(y_{\epsilon}, s_{\epsilon}\right)$, which is bigger than that of $\Gamma(W)$ at $\left(y_{\epsilon}, s_{\epsilon}\right)$. By arguing as in the previous step, one can easily see that the slope of $\Gamma(W)$ at $\left(y_{\epsilon}, s_{\epsilon}\right)$ is bigger than $n-1$ at $\left(s_{\epsilon}, y_{\epsilon}\right)$ for small $\epsilon>0$ where $n$ is the slope of $\Gamma(W)$ at $\left(x_{0}, t_{0}\right)$. Hence for small $\epsilon>0$ we have

$$
n-2 \leq b_{\epsilon} /\left|q_{\epsilon}\right| \leq a_{\epsilon} /\left|p_{\epsilon}\right| \leq m+1 \text {. }
$$

Hence we can 'tilt' $B_{2}^{\epsilon}$ : namely, we consider $\tilde{B}_{2}^{\epsilon}$ : a space-time ball so that $\tilde{B}_{2}^{\epsilon}=B_{2}^{\epsilon}$ at $t=t_{\epsilon}^{1}$ and the slope of $\tilde{B}_{2}^{\epsilon}$ at $\left(x_{\epsilon}^{1}, t_{\epsilon}^{1}\right)$ is smaller than that of $\Gamma\left(\varphi^{\epsilon}\right)$ at the point by $\epsilon^{1 / 4}$. (This is possible because $a_{\epsilon} /\left|p_{\epsilon}\right|$ is bounded from below due to (2.10).)

Lemma 2.2 For each $\epsilon>0$ There is a hypersurface $\Sigma^{\epsilon}=\partial S^{\epsilon}$ in $\mathbb{R}^{n+1}$ such that 


$$
\left\{\begin{array}{l}
S^{\epsilon} \subset \tilde{B}_{2}^{\epsilon} \cap\left\{t \leq t_{\epsilon}^{1}\right\}, \quad\left(x_{\epsilon}^{1}, t_{\epsilon}^{1}\right) \in \Sigma^{\epsilon} \\
\Sigma^{\epsilon} \text { is locally Lipschitz in space and time } \\
\Sigma^{\epsilon} \subset \Omega(U)^{C}
\end{array}\right.
$$

Moreover at $\left(x_{\epsilon}^{1}, t_{\epsilon}^{1}\right) \Sigma^{\epsilon}$ has the same mean curvature and slower advancing speed by $\epsilon^{1 / 4}$ compared to those of $\Gamma\left(\varphi^{\epsilon}\right)$ at $\left(x_{\epsilon}, t_{\epsilon}\right)$.

\section{Proof}

1. From the construction of $\varphi^{\epsilon}$, there is $h>0$ such that $\varphi^{\epsilon} \geq \bar{Z}$ in a cylindrical domain $N=D_{h}\left(x_{\epsilon}\right) \times\left[t_{\epsilon}-h, t_{\epsilon}\right]$ (We may pick $h=o(\epsilon)$ for later use.)

On the other hand $\Gamma\left(\phi^{\epsilon}\right)$ (locally) lies in $\bar{\Omega}(W)$ and touches $\Gamma(W)$ at $\left(y_{\epsilon}, s_{\epsilon}\right)$ from inside. Thus it follows that

$$
\kappa_{\left(y_{\epsilon}, s_{\epsilon}\right)}\left(\Gamma\left(\phi^{\epsilon}\right)\right) \geq \kappa_{\left(y_{\epsilon}, s_{\epsilon}\right)}(\Gamma(W)) \geq-C / r, \quad C=C(r)>1 .
$$

where the last inequality is due to Lemma 1.3. Also due to (2.9)(iii) we have

$$
X_{\epsilon} /\left|p_{\epsilon}\right|-Y_{\epsilon} /\left|q_{\epsilon}\right| \leq \epsilon I
$$

and from above two inequalities it follows that

$$
\kappa_{x_{\epsilon}}\left(\Gamma_{t_{\epsilon}}\left(\varphi^{\epsilon}\right)\right) \geq \kappa_{y_{\epsilon}}\left(\Gamma_{s_{\epsilon}}\left(\phi^{\epsilon}\right)\right) \geq-C / r+O(\epsilon)
$$

2. Hence if we choose $C$ in the defition of $B_{\epsilon}^{2}$ the same as that in (2.13), after a translation of $\left(x_{\epsilon}, t_{\epsilon}\right)$ to $\left(x_{\epsilon}^{1}, t_{\epsilon}^{1}\right), \Gamma\left(\varphi^{\epsilon}\right)$ lies outside of $B_{2}$ in $D_{h}\left(x_{\epsilon}^{1}\right)$ if $h<<r$ (see figure 5.) Let us denote $\Gamma^{\prime}\left(\varphi^{\epsilon}\right)$ as the translated version of $\Gamma\left(\varphi^{\epsilon}\right)$.

3. Let us divide $D_{2}^{\epsilon}$ into two halves $D_{2}^{\epsilon, \pm}$ cutting by the hypersurface normal to $e_{n}$, where $D_{2}^{\epsilon,+}$ is the one closer to $\left(x_{\epsilon}^{1}, t_{\epsilon}^{1}\right)$. Now we consider $\Gamma_{1}\left(t_{\epsilon}^{1}\right)=\Gamma_{t_{\epsilon}^{1}}^{\prime}\left(\varphi^{\epsilon}\right) \cap\left\{\left|x^{\prime}\right|=h\right\}$ and $\Gamma_{2}\left(t_{\epsilon}^{1}\right)=D_{2}^{\epsilon,+} \cap\left\{\left|x^{\prime}\right|=h\right\}$ (see figure 5.)

We consider

$$
S\left(t_{1}^{\epsilon}\right)=\left\{s x+(1-s) y: x \in \Gamma_{1}\left(t_{\epsilon}^{1}\right), y \in \Gamma_{2}\left(t_{\epsilon}^{1}\right), s \in[0,1] .\right\}
$$




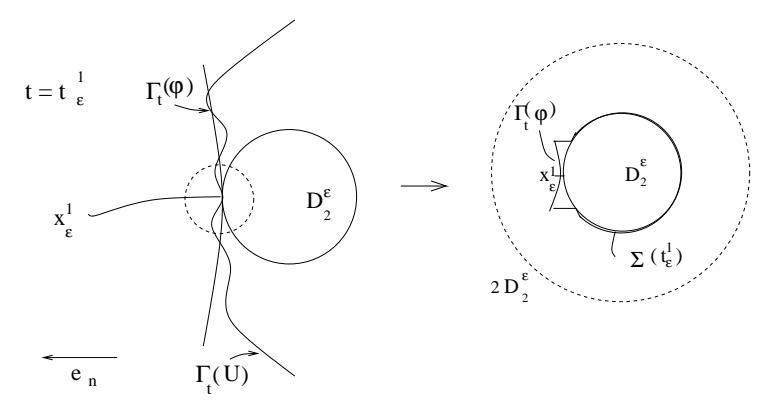

Figure 5.

Then the region surrounded by

$$
\Sigma\left(t_{1}^{\epsilon}\right)=\left[\Gamma_{t_{\epsilon}^{1}}^{\prime}(\varphi) \cap\left\{\left|x^{\prime}\right| \leq h / 2\right\}\right] \cup S\left(t_{\epsilon}^{1}\right) \cup\left[D_{2}^{\epsilon,+} \cap\left\{\left|x^{\prime}\right|>h\right\}\right] \cup D_{2}^{\epsilon,-} .
$$

includes $D_{2}^{\epsilon}$.

4. Next for $t \in\left[t_{\epsilon}^{1}-h, t_{\epsilon}^{1}\right)$, we translate $\Gamma_{t}^{\prime}\left(\varphi^{\epsilon}\right)$ toward the $-e_{n}$ direction so that $\Gamma_{t}^{\prime}\left(\varphi^{\epsilon}\right)$ touches $\tilde{B}_{2}^{\epsilon}$ at a point. Let us denote $\Gamma_{t}^{\prime \prime}\left(\varphi^{\epsilon}\right)$ as the translated version of $\Gamma_{t}^{\prime}\left(\varphi^{\epsilon}\right)$, and let $h(t)=\left(t-t_{\epsilon}^{1}+h\right)$. We then proceed as in the previous step to connect $\Gamma_{1}(t)=\Gamma_{t}^{\prime \prime}\left(\varphi^{\epsilon}\right) \cap\left\{\left|x^{\prime}\right|=h(t) / 2\right\}$ and $\Gamma_{2}(t)=\tilde{D}^{\epsilon_{2},+}(t) \cap\left\{\left|x^{\prime}\right|=h(t)\right\}$ where $\tilde{D}_{2}^{\epsilon}(s)=\tilde{B}_{2}^{\epsilon} \cap\{t=s\}$. Then the region surrounded by

$$
\Sigma=\left\{(x, t): x \in \Sigma(t), t \in\left[t_{\epsilon}^{1}-h, t_{\epsilon}^{1}\right]\right\}
$$

includes $\tilde{B}_{2}^{\epsilon} \cap\left[t_{\epsilon}^{1}-h, t_{\epsilon}^{1}\right]$ and $\Sigma=\Gamma^{\prime \prime}$ in a small parabolic neighborhood of $\left(x_{\epsilon}^{1}, t_{\epsilon}^{1}\right)$.

Note that $\Sigma\left(t_{\epsilon}^{1}-h\right)=\tilde{B}_{2}^{\epsilon} \cap\left\{t=t_{\epsilon}^{1}-h\right\}$, and hence we can extend $\Sigma$ by defining $\Sigma=\tilde{B}_{2}^{\epsilon}$ for $t_{\epsilon}^{2} \leq t \leq t_{\epsilon}^{1}-h$, where $t_{\epsilon}^{2}$ is chosen such that $\tilde{D}_{2}\left(t_{\epsilon}^{2}\right)$ has a positive radius. (Note that $t_{\epsilon}^{1}-t_{\epsilon}^{2}$ depends only on $r$.)

Now for each $\epsilon>0$ we have constructed a hypersurface $\Sigma\left(=\Sigma^{\epsilon}\right)$ of size independent of $\epsilon>0$, which lies outside of $\Omega(U)$ and coincides with $\Gamma^{\prime \prime}(\varphi)$ in a parabolic neighborhood $\tilde{N}$ of $\left(x_{\epsilon}^{1}, t_{\epsilon}^{1}\right)$. Moreover it follows from the construction that $\Sigma$ is locally representable as a Lipschitz continuous graph in space-time.

Next we construct a local barrier function based on $\Sigma^{\epsilon}$.

\section{Constructing the barrier function}

Consider a space-time ball $B_{3}^{\epsilon}=(1+\delta) \tilde{B}_{2}^{\epsilon}$, where $\delta=O\left(\epsilon^{1 / 3}\right)$. We consider a solution $\psi$ of the Stefan problem in the region $\Pi=\left(B_{3}^{\epsilon}-\Sigma\right) \cap\left[t_{\epsilon}^{2}, t_{\epsilon}^{1}\right]$ with $\psi=0$ on $\Sigma$ and $\psi=M$ on $\partial B_{3}^{\epsilon}$. If 
we choose the initial data to be harmonic in $\Pi \cap\left\{t=t_{2}^{\epsilon}\right\}$, then we obtain a unique function $\psi$ which is continuous up to $\bar{\Pi}$ and smooth up to $\bar{\Pi}$ in $\tilde{N}$. Moreover by comparing with a solution $\tilde{\psi}(x, t)$ of the Stefan problem in the region $\left(B_{3}^{\epsilon}-\tilde{B}_{2}^{\epsilon}\right) \cap\left[t_{2}^{\epsilon}, t_{1}^{\epsilon}\right]$ with $\tilde{\psi}=0$ on $\partial \tilde{B}_{2}^{\epsilon}$ and $\tilde{\psi}=M$ on $\partial B_{3}^{\epsilon}$ and $\tilde{\psi}=\psi$ on $t=t_{2}^{\epsilon}$, we obtain that

$$
|D \psi|\left(x_{\epsilon}^{1}, t_{\epsilon}^{1}\right)=e_{n} \cdot D \psi\left(x_{\epsilon}^{1}, t_{\epsilon}^{1}\right) \leq|D \tilde{\psi}|\left(x_{\epsilon}^{1}, t_{\epsilon}^{1}\right) \leq \frac{M}{r_{1} \delta}(1+O(\delta))
$$

where $r_{1}$ is the radius of $\tilde{B}_{2}^{\epsilon} \cap\left\{t=t_{\epsilon}^{1}\right\}$.

Lemma 2.3 We can choose $M$ in the definition of $\psi$ such that $U \leq \psi$ in $\Pi$ and

$$
|D \psi| \leq \frac{Z\left(x_{\epsilon}+\delta_{1} r_{1} e_{n}^{\prime}\right)}{r_{1} \delta_{1}}\left(1+O\left(\delta_{1}\right)\right)
$$

where $e_{n}^{\prime}:=\left(y_{\epsilon}-x_{\epsilon}\right) / \delta_{1} r_{1}+q_{\epsilon} /\left|q_{\epsilon}\right|$

1. We compare $U$ and $\psi$ in $\Pi$. From the construction of $\tilde{B}_{2}^{\epsilon}$, it follows that $B_{3}^{\epsilon} \cap\left\{t=t_{2}^{\epsilon}\right\}$ will lie outside of $\Omega(U)$ since $\delta<<\epsilon^{1 / 4}$, and thus $U=0 \leq \psi$ at $t=t_{2}^{\epsilon}$. Since $U=0 \leq \psi$ on $\Sigma$, if we choose $M>0$ such that $U \leq M$ on $\partial B_{3}^{\epsilon}$, then it follows that $U \leq \psi$ in $\Pi$.

2. Now we proceed and choose $M>0$ for the construction of $\psi$. Let $\delta_{1}=O\left(\epsilon^{1 / 3}\right)$. Observe that by definition of $Z, U \leq Z\left(x_{\epsilon}+\delta_{1} r_{1} e_{n}^{\prime}, t_{\epsilon}\right)$ on $B_{r}\left(x_{\epsilon}+\delta_{1} r_{1} e_{n}^{\prime}\right)$ for any vector $e_{n}^{\prime}$ in $\mathbb{R}^{n}$. We choose

$$
e_{n}^{\prime}:=\left(y_{\epsilon}-x_{\epsilon}\right) / \delta_{1} r_{1}+q_{\epsilon} /\left|q_{\epsilon}\right|=q_{\epsilon} /\left|q_{\epsilon}\right|+O\left(\epsilon^{1 / 6}\right)=e_{n}+O\left(\epsilon^{1 / 6}\right)
$$

( The first equality holds since $\left|x_{\epsilon}-y_{\epsilon}\right|=O\left(\epsilon^{1 / 2}\right)$ and the second holds since $\left|p_{\epsilon} /\right| p_{\epsilon}\left|-q_{\epsilon} /\right| q_{\epsilon}||=O(\epsilon)$ due to (2.7) (iii).)

3. Since $\tilde{B}_{2}^{\epsilon} \cap\left[t_{\epsilon}^{2}, t_{\epsilon}^{1}\right] \subset B_{r}\left(x_{\epsilon}\right)$ and $\tilde{B}_{2}^{\epsilon}$ has radius strictly smaller than $r$, for sufficiently small $\epsilon>0$ we have $\left(1+\delta_{1}\right) \tilde{B}_{2}^{\epsilon} \subset B_{r}\left(x_{\epsilon}+\delta_{1} r_{1} e_{n}\right)$ in $\left[t_{\epsilon}^{2}, t_{\epsilon}^{1}\right]$.

Since $B_{r}\left(x_{\epsilon}+\delta_{1} r_{1} e_{n}\right)$ is at most $\delta_{1} r_{1}\left|e_{n}-e_{n}^{\prime}\right|$ away from $B_{r}\left(x_{\epsilon}+\delta_{1} r_{1} e_{n}^{\prime}\right)$, it follows that $\left(1+\delta_{1}-\sigma\right) \tilde{B}_{2}^{\epsilon} \subset B_{r}\left(x_{\epsilon}+\delta_{1} r_{1} e_{n}^{\prime}\right)$ where $\sigma=\delta_{1}\left(1-e_{n} \cdot e_{n}^{\prime}\right)=o\left(\delta_{1}\right)$. Thus if we let $M$ be the supremum of $U$ in $\left(1+\delta_{1}-\sigma\right) \tilde{B}_{2}^{\epsilon}$ and consider $\delta=\delta_{1}-\sigma$ in the construction of $\psi$ in step 1 . then $M \leq Z\left(x_{\epsilon}+\delta_{1} r_{1} e_{n}^{\prime}, t_{\epsilon}\right), U \leq M$ on $\partial B_{3}^{\epsilon}$ and

$$
U \leq \psi \text { in } \Pi, \quad|D \psi|\left(x_{\epsilon}^{1}, t_{\epsilon}^{1}\right) \leq \frac{M}{r_{1} \delta_{1}}\left(1+O\left(\delta_{1}\right)\right) .
$$


4. Observe that $M / \delta_{1} r_{1}$ is bounded independent of $\epsilon>0$. To see this let us we consider a solution $\chi$ of the heat equation in $D=\left(2 \tilde{B}_{2}^{\epsilon}-\tilde{B}_{2}^{\epsilon}\right) \cap\left[t_{\epsilon}^{2}, t_{\epsilon}^{1}\right]$ with

$$
\begin{cases}\chi=S=\sup _{2 B_{2}^{\epsilon}} U & \text { on } \partial\left(2 B_{2}^{\epsilon}\right) \\ \chi=0 & \text { on } \partial \tilde{B}_{2}^{\epsilon} \\ \chi\left(x, t_{\epsilon}^{2}\right)=S & \text { in } 2 \tilde{B}_{2}^{\epsilon}-B_{2}^{\epsilon} \\ -\Delta \chi\left(x, t_{\epsilon}^{2}\right)=0 & \text { in } B_{2}^{\epsilon}-\tilde{B}_{2}^{\epsilon}\end{cases}
$$

Then $U \leq \chi$ in $D$ and hence $M$ is less than the supremum of $\chi$ in $(1+\delta) B_{3}^{\epsilon}$, which is of size $r_{1} \delta_{1}|D \chi|\left(x_{\epsilon}^{1}, t_{\epsilon}^{1}\right)+o(\delta)|D \chi|$. Note that $|D \chi|$ is bounded, whose bound depend only on $r$ and $S$. (We used the fact that $D$ is a domain of size only depending on $r$.)

Hence $M / r_{1} \delta_{1} \leq|D \chi|+o(1)$.

Finally we are ready for proving the main theorem.

\section{Proof of theorem 1.2}

1. Applying a symmetric argument as above to $\phi^{\epsilon}$ and $V$, one can construct a domain $\Pi_{2}$ which coincides with a translate of $\Gamma\left(\phi^{\epsilon}\right)$ in a small neighborhood of $\left(y_{\epsilon}^{1}, s_{\epsilon}^{1}\right) \in \Gamma(V)$ and a barrier function $\xi$ in $\Pi_{2}$ such that for $\delta_{2}=O\left(\epsilon^{1 / 3}\right)$

$$
V \geq \xi \text { in } \Pi_{2}, \quad|D \xi|\left(y_{\epsilon}^{1}, s_{\epsilon}^{1}\right) \geq \frac{W\left(y_{\epsilon}+\delta_{2} r \frac{q_{\epsilon}}{\left|q_{\epsilon}\right|}, s_{\epsilon}\right)}{\delta_{2} r}\left(1+O\left(\delta_{2}\right)\right)
$$

2. By the definition of $U$ and $V$ we have at $\left(x_{\epsilon}^{1}, t_{\epsilon}^{1}\right)$

$$
\psi_{t} /|D \psi|-|D \psi|+\kappa_{1} \leq 0
$$

and at $\left(y_{\epsilon}^{1}, s_{\epsilon}^{1}\right)$

$$
\xi_{t} /|D \xi|-|D \xi|+\kappa_{2}-\gamma \geq 0
$$

where $\kappa_{1}$ is the mean curvature of $\Gamma_{t_{\epsilon}^{1}}(\psi)$ at $x_{\epsilon}^{1}$ and $\kappa_{2}$ is that of $\Gamma_{s_{\epsilon}^{1}}(\xi)$ at $y_{\epsilon}^{1}$. But from the construction of $\psi$ and $\xi, \kappa_{1}$ is the mean curvature of $\Gamma_{t_{\epsilon}}\left(\varphi^{\epsilon}\right)$ at $x_{\epsilon}$ and $\kappa_{2}$ is that of $\Gamma_{s_{\epsilon}}(\phi)$ at $y_{\epsilon}$. Hence due to (2.13) 


$$
\kappa_{1} \geq \kappa_{2}+O(\epsilon)
$$

Furthermore $\psi_{t} /|D \psi|\left(x_{\epsilon}^{1}, t_{\epsilon}^{1}\right)$ equals the slope of $\Gamma(\varphi)$ at $\left(x_{\epsilon}, t_{\epsilon}\right)$, which is equal to $a_{\epsilon} /\left|p_{\epsilon}\right|-\epsilon^{1 / 4}$, and similarly $\xi_{t} /|D \xi|\left(y_{\epsilon}^{1}, s_{\epsilon}^{1}\right)=b_{\epsilon} /\left|q_{\epsilon}\right|+\epsilon^{1 / 4}$. Due to (2.8), (2.9) (iii) and (2.11),

$$
a_{\epsilon} /\left|p_{\epsilon}\right| \geq b_{\epsilon} /\left|q_{\epsilon}\right|(1+O(\epsilon)) \geq b_{\epsilon} /\left|q_{\epsilon}\right|+O(\epsilon) .
$$

Therefore subtracting the second line from the first and applying (2.17)-(2.18), we obtain

$$
|D \xi|\left(x_{\epsilon}^{1}, t_{\epsilon}^{1}\right)-|D \psi|\left(y_{\epsilon}^{1}, s_{\epsilon}^{1}\right)+\gamma \leq O(\epsilon)+2 \epsilon^{1 / 4} .
$$

Hence to obtain a contradiction, it is enough to show that

$$
|D \psi|\left(y_{\epsilon}^{1}, s_{\epsilon}^{1}\right) \leq|D \xi|\left(x_{\epsilon}^{1}, t_{\epsilon}^{1}\right)+o(1) .
$$

3. By the definition of $e_{n}^{\prime}$, we have $y_{\epsilon}+\delta_{1} r_{1} q_{\epsilon} /\left|q_{\epsilon}\right|=x_{\epsilon}+\delta_{1} r_{1} e_{n}^{\prime}$. Since

$Z\left(\cdot, t_{\epsilon}\right) \leq W\left(\cdot, s_{\epsilon}\right)+O\left(\epsilon^{2}\right),((2.9)(\mathrm{v}))$, if we choose $\delta_{2}$ such that $\delta_{2} r=\delta_{1} r_{1}$ it follows from (2.14)-(2.16) that

$$
|D \psi|\left(y_{\epsilon}^{1}, s_{\epsilon}^{1}\right)-|D \xi|\left(x_{\epsilon}^{1}, t_{\epsilon}^{1}\right) \leq O(\delta) M / r_{1} \delta_{1}+O\left(\epsilon^{2}\right) / r_{1} \delta_{1}
$$

Since $\delta_{1}=O\left(\epsilon^{1 / 3}\right)$ and $M / r_{1} \delta_{1}$ is uniformly bounded independent of $\epsilon>0$ (shown below (2.15)), we can conclude.

\section{$3 \quad$ Existence and Uniqueness}

Theorem 3.1 For a given initial data $u_{0} \in C^{+}(\mathbb{R})$ with compact support, there exist the minimal and maximal viscosity solution of $(P)$ with initial data $u_{0}$.

\section{Proof}

1. We apply the Perron's method, which is often used to prove the existence of viscosity solutions. The main step for this method is to construct a viscosity sub- and supersolution of (P) with given initial data $u_{0}$. 
2. We first construct a subsolution of $(\mathrm{P})$. Let $\alpha_{0} \geq 0$ be the unique continuous viscosity solution of the motion by mean curvature

$$
V=-\kappa,
$$

with initial data $u_{0}$ (see [ES], [ESS] for comparison principle and further properties of viscosity solutions of $(\mathrm{MC})$.) For $(x, t) \in \bar{\Omega}\left(\alpha_{0}\right)$, let us define

$$
\alpha(x, t)=\sup \left\{\gamma(x, t): \gamma_{t}-\Delta \gamma \leq 0 \text { in } \Omega\left(\alpha_{0}\right) \text { with } \gamma=0 \text { on } \Gamma\left(\alpha_{0}\right),\right\}
$$

and let us define $\alpha$ to be zero elsewhere. Then $\alpha^{*}$ is a viscosity subsolution of $(\mathrm{P})$ with $\bar{\Omega}\left(\alpha^{*}\right)=\bar{\Omega}\left(\alpha_{0}\right)$.

Moreover $\alpha$ satisfies

$$
\alpha^{*}(x, 0)=\alpha_{*}(x, 0)=u_{0}(x)
$$

In fact, if $\beta_{0}(x, 0)$ is a smooth initial data with $\left|D \beta_{0}(x, 0)\right| \neq 0$ on $\Gamma\left(\beta_{0}(x, 0)\right)$ and $u_{0} \prec$ $\beta_{0}(x, 0)$, then due to the comparison principle of (MC) $\beta_{0}(x, t)$ : the corresponding solution of (MC) satisfies $\alpha_{0} \leq \beta_{0}$. Moreover by the short-time existence result of (MC) (see [ES]), $\beta_{0}$ and $\Gamma\left(\beta_{0}\right)$ is smooth for small time. Therefore $\beta$, correspondingly defined as $\alpha$ in (3.1), is smooth for small time and $\alpha^{*} \leq \beta$. It follows from this argument that $\alpha^{*}(x, 0) \leq u_{0}(x)$ and $\bar{\Omega}\left(\alpha^{*}\right) \cap\{t=0\}=\bar{\Omega}\left(u_{0}\right)$. It also follows from a parallel argument that $\alpha_{*}(x, 0) \geq u_{0}(x)$. Therefore (3.2) holds and $\alpha^{*}$ is a viscosity subsolution of $(P)$ with initial data $u_{0}$.

3. Next we construct $\gamma$ : a supersolution of $(\mathrm{P})$ with initial data $u_{0}$. We let $\gamma(x, t)$ solve the heat equation with initial data $u_{0}$. Note that $\gamma$ is continuous. Now we consider

$$
U(x, t)=\sup \left\{h(x, t): \text { a visc. subsolution of }(\mathrm{P}), h(x, 0)=u_{0}(x) \text { and } \alpha^{*} \leq h .\right\}
$$

Then $U^{*}$ satisfies (3) of Definition 1.1 (a) and $U^{*} \leq \gamma^{*}=\gamma$, and thus $U^{*}(x, 0)=u_{0}(x, 0)$. Moreover by a barrier argument, one can easily show that $\bar{\Omega}\left(U^{*}\right) \cap\{t=0\}=\bar{\Omega}\left(u_{0}\right)$. Hence $U^{*}$ is a subsolution of $(\mathrm{P})$ with initial data $u_{0}$ and hence $U=U^{*}$ by definition.

4. Due to (3.2), it follows that $U_{*}(x, t)=u_{0}(x)$ and $\overline{\Omega\left(U_{*}\right) \cap\{t>0\}} \cap\{t=0\}=\bar{\Omega}\left(u_{0}\right)$. Now one can proceed as in the proof of Theorem 4.7 in [K1] to show that $U_{*}$ satisfies (3) of Definition 1.1 (b) and therefore $U_{*}$ is a supersolution of $(P)$ with initial data $u_{0}$. 
5. Now consider $v$ : a viscosity solution of $(\mathrm{P})$ with initial data $u_{0}$. If we approximate $u_{0}$ by smooth initial data $\beta_{0}^{\epsilon}(x, 0) \prec u_{0}$ which increases as $\epsilon \rightarrow 0$ and let $\beta_{0}^{\epsilon}(x, t)$ be the solution of (MC) with initial data $\beta_{0}^{\epsilon}(x, 0)$, then by comparison principle of (MC) $\beta_{0}^{\epsilon}$ is increasing with respect to $\epsilon$ at any fixed $(x, t)$. Moreover $\beta_{0}(x, t):=\left(\lim _{\epsilon \rightarrow 0} \beta_{0}^{\epsilon}(x, t)\right)_{*}$ is a supersolution of (MC) with initial data $u_{0}$ by the stability property of the viscosity solutions. Hence by the comparison principle of (MC) $\alpha_{0}=\beta_{0}$ and $\Gamma\left(\beta_{0}^{\epsilon}\right)$ converges to $\Gamma\left(\alpha_{0}\right)$. Hence if we define $\beta^{\epsilon}$ as in (3.1) with $\beta_{0}^{\epsilon}$, then we have

$$
\alpha_{*} \leq \lim _{\epsilon \rightarrow 0} \beta^{\epsilon}
$$

by the stability property of the heat equation with respect to the domain variance. On the other hand $\beta^{\epsilon} \leq v$ due to Theorem 1.2 , and hence it follows from (3.3) that $\alpha^{*} \leq v^{*}$.

It follows that $v^{*} \leq U$ by definition of $U$ and hence $U$ is the maximal viscosity solution of $(\mathrm{P})$.

6. Similarly one can show that

$$
V(x, t)=\inf \left\{h(x, t): \text { a viscosity supersolution of }(\mathrm{P}), h(x, 0)=u_{0} \text { and } h \leq \gamma\right\}
$$

is the minimal viscosity solution of $(\mathrm{P})$.

Next we show that there is a unique solution of $(\mathrm{P})$ when the initial data satisfies several additional conditions.

Theorem 3.2 For $u_{0} \in C^{+}(\mathbb{R})$ with compact support, suppose that either (i) $u_{0}$ is radially monotone, that is $u_{0}(x) \prec u_{0}(x / 1+\epsilon)$ for any small $\epsilon>0$ or (ii) $\Gamma\left(u_{0}\right)$ is smooth and $u_{0}$ is strictly concave in $\bar{\Omega}\left(u_{0}\right)$ with $\left|D u_{0}\right|(x)<\kappa_{x}\left(\Gamma\left(u_{0}\right)\right)$ on $\Gamma\left(u_{0}\right)$. Then there is a unique viscosity solution $u$ of $(P)$ with initial data $u_{0}$.

\section{Proof.}

Let $u$ and $v$ be viscosity solutions of $(\mathrm{P})$ with initial data $u_{0}$. In case of (i), observe that

$$
v_{\epsilon}(x, t):=v\left(x /(1+\epsilon), t /(1+\epsilon)^{2}\right)
$$

is a viscosity supersolution of $(\mathrm{P})$ with $u(x, 0) \prec v_{\epsilon}(x, 0)$. Thus Theorem 1.2 yields that $u \prec v_{\epsilon}$ for any small $\epsilon>0$, and thus $u=u_{*} \leq v=v_{*}$. Similarly it follows that $v \leq u$ and the uniqueness follows. 
In case of (ii) it is easy to check by a barrier argument that $v$ decreases at $t=0$ since, formally speaking, $v_{t}(x, 0)=\Delta u_{0}(x, 0)<0$ and $V=|D u|-\kappa<0$ on $\Gamma\left(u_{0}\right)$. Thus $v(x, \epsilon) \prec u_{0}(x)$ for small $\epsilon>0$. Now one can apply Theorem 1.2 to

$$
v_{\epsilon}(x, t)=v(x, t+\epsilon) \text { and } u(x, t)
$$

and proceed as in case (i).

\section{Remark}

We also expect the uniqueness result to hold for directionally monotone solutions in cylindrical domains with Neumann boundary condition on the boundary of the domain. We refer to [K2] where the assertion is proved for the flame propagation model

$$
\begin{cases}u_{t}-\Delta u=0 & \text { in }\{u>0\}, \\ |D u|=1 & \text { on } \partial\{u>0\} .\end{cases}
$$

\section{The problem of nonuniqueness}

In general uniqueness of the solution is not expected, as the following example in $\mathbb{R}^{2}$ indicates. Consider radial symmetric initial data $u_{0}$ in the form of a hump with compact support and a bell-shaped form. If the initial gradient is larger than the mean curvature of the free boundary than the support of the solution of $(\mathrm{P})$ with initial data $u_{0}$ begins its evolution by expanding. However one can easily check with barrier functions - for example one can use the self-similar solutions of $(\mathrm{FP})$ given in $[\mathrm{V}]$ - that there is a time $t_{0}$ where the free boundary radius begins shrinking for the first time. Let $r_{0}$ be the free boundary radius at $t=t_{0}$.

Now if we start with two humps, that is if we consider the initial data

$$
v(x)=\max \left[u_{0}(x), u_{0}\left(x+x_{1} e_{1}\right)\right]
$$

where $e_{1}=(1,0)$ and $x_{1}=2 r_{0}$, then the solution of $(\mathrm{P})$ with the initial data $u_{0}$ has two separate components for its support till they contact each other at $t=t_{0}$ (see figure 6.) Now if we superpose the separate evolution of the two humps for $t>t_{0}$, then the support separate and begin shrinking in the form of two separate balls at least for a small time.

However if we consider the initial data with $x_{1}<2 r_{0}$, then the supports contact at a point $x_{0}$ at $t=t_{1}<t_{0}$ when the supports are still expanding. In this case the supports must merge into one after $t>t_{1}$. Moreover we expect the narrow neck formed near $x_{0}$ for $t>t_{1}$ to expand out very quickly due to the mean curvature term in the free boundary motion law in $(\mathrm{P})$. Indeed the concave part of the merged free boundary will keep expanding until it becomes convex. Thus if we take the limit $x_{1} \rightarrow 2 r_{0}$, it is conceivable that we will get a different solution than the simple superposition discussed above. 


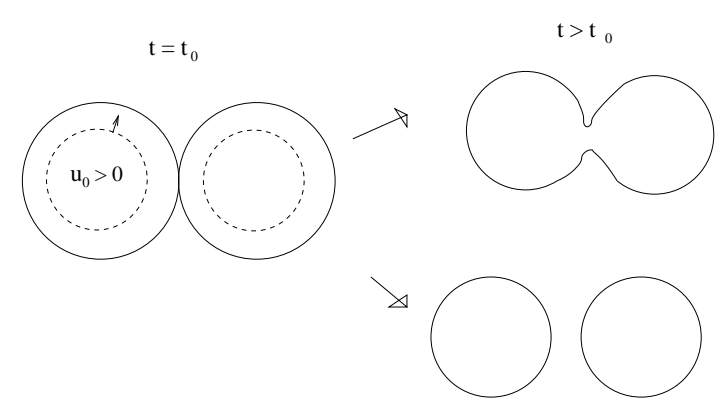

Figure 6.

\section{References}

[ACS] I. Athanasopoulos, L.A. Caffarelli and S. Salsa, Stefan-like problems with curvature, J. Geom. Anal. 13 (2003) 21-27.

[ACKS] I. Athanasopoulos, L.A. Caffarelli,C. Kenig and S. Salsa, An area-Dirichlet integral minimization problem, Comm. Pure Appl. Math 54 (2001) 479-499.

[CIL] M.G. Crandall, H. Ishii, and P.L. Lions, User's guide to viscosity solutions of secondorder partial differential equations, Bull.Amer.Math.Sc. 27, 1(1992), 1-67.

[CL] M.G. Crandall and P.L. Lions, Viscosity solutions of Hamilton-Jacobi eqations, Trans. Amer. Math. Soc. 277 (1983), 1-42.

[ES] L.C. Evans and J. Spruck, Motion of level sets by mean curvature I, J. Diff. Geom.33(1991) 635-681.

[ESS] L.C. Evans, H.M. Soner and P.E. Souganidis, Phase transitions and generalized motion by mean curvature, Comm.Pure Appl.Math. 45(1992) 1097-1123.

[K1] I.C. Kim, Uniqueness and Existence results on viscosity solutions of the Hele-Shaw and the Stefan problems, Arch. Rat. Mech. Anal.168 (2003) 299-328.

[K2] I.C. Kim, A free boundary problem arising in flame propagation, J. Diff. Equations, 191, (2003) 470-489

[V] J.L. Vazquez, The free boundary problem for the heat equation with fixed gradient condition, Free boundary problems, theory and applications (Zakopane, 1995), 277-302, Pitman Res. Notes Math. Ser. 363, Longman, Harlow, 1996. 